\title{
Automatic Fingerprint Identification System Using Robust Distance
}

\author{
Wasfi T. Saalih \\ Statistics Department \\ College of Administration \& Economics \\ Salahaddin University \\ wasfit@yahoo.com
}

\author{
Dilshad Sh. E. Botani \\ Mathematics Department \\ College of Science \\ Salahaddin University \\ Dilshadss2004@yahoo.com
}

\begin{abstract}
Fingerprint recognition has attracted many researchers in order to conduct studies in this field. The need for finding an AFIS (Automatic Fingerprints Identification System) has been an interesting subject by scientists, bureaus and companies, because the increasing use of AFIS leads to solve many problems of identification, because there are no tools or machines in the region except classical methods.

The fingerprint department at the Criminal Identification Bureau in Erbil uses habitually very classical methods to identify the fingerprints. There are (8000) fingerprints in that department related to criminals.

To create an AFIS, we depended on the stages of image analysis using Visual Basic. These stages are very essential to obtain an excellent system for fingerprints recognition. The system begins with acquiring fingerprint images of (BMP, JPEG, GIF, DIB) types and stretching (shrinking) them to $(200 \times 200)$ pixels.

The study deals with a new procedure to segment the fingerprint image via dividing the fingerprint to 64 squares, the size of each square is equal to $(20 \times 20)$ pixels. Then, we used a new procedure to describe each square (inside the image) after representing the fingerprint image by its grayscales. Eight parameters were used to describe the squares: Mean, Median, Quadratic mean, Harmonic mean, Variance, Skewness, Kurtosis and Mean deviation. A robust measure for finding the minimum distances between the squares of different fingerprints was used to the recognition process and applying professional systems to perform the interpretation step to reach the end of this process, and we achieved $(\mathbf{8 4 . 5} \%)$ success.
\end{abstract}

$$
\text { نظام آلي لتشخيص طبعاث الأصسابع باستخدام المسافات الحصينة }
$$

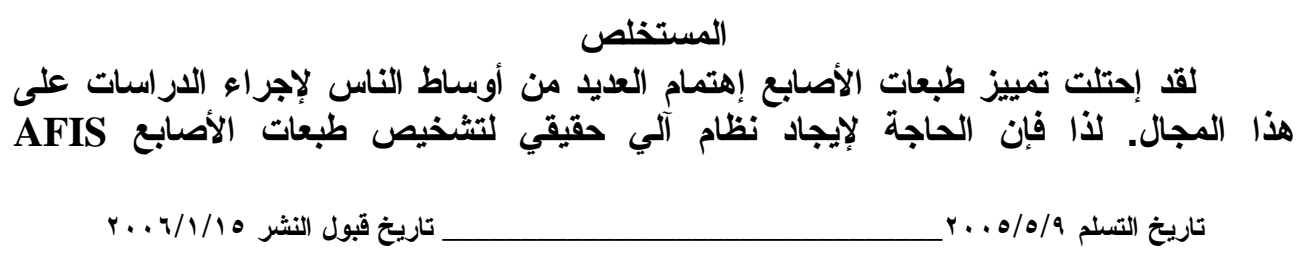




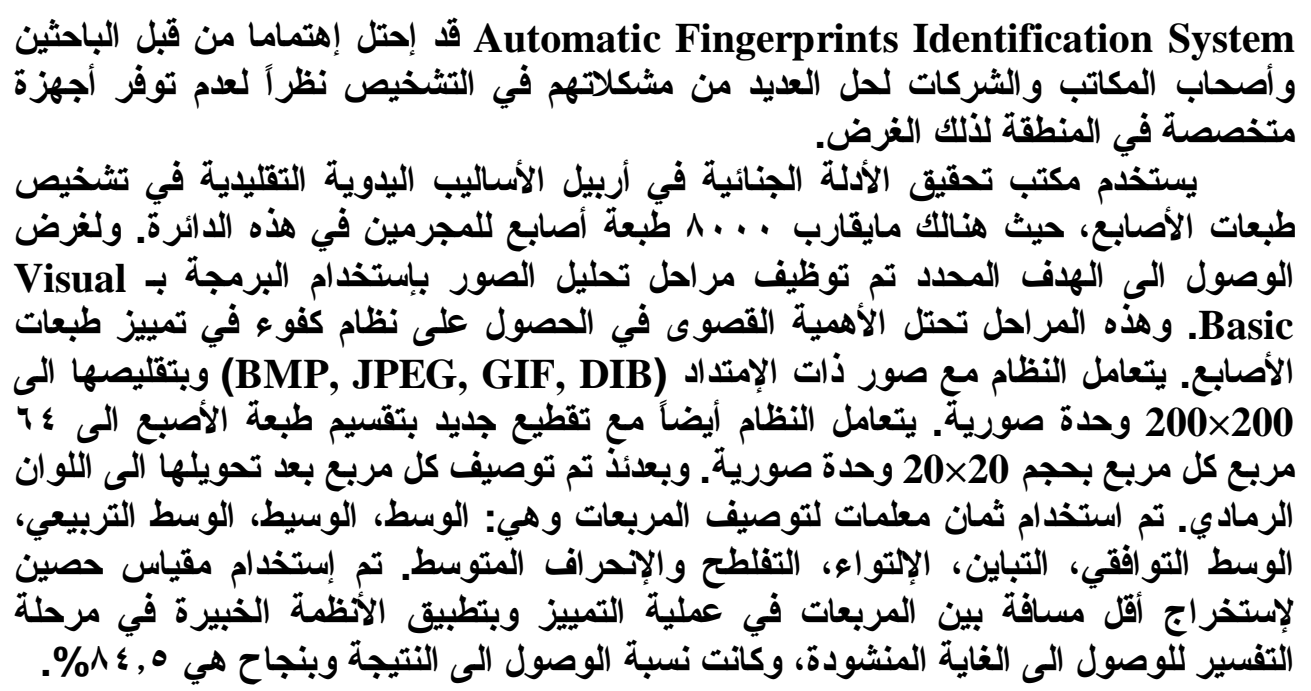

\section{Introduction}

In the past decade, the interest in fingerprint-based biometrics systems has been increased significantly [14]. Fingerprints are considered as an important device to the personal identification. Although, there are many other unique characteristics of an individual that can be used such as voiceprints, dental impressions, DNA, Retinal Patterns, Iris Patterns, Face Patterns, and even the shape of the Ear Lobes [3]. They can by each of this Biometrics( $\left(^{*}\right)$ identifications recognize the individuals, but each one has its own merits and uses depending on the place and time of occurring.

In 1915, the idea of fingerprint had entered Iraq then some Indian and English experts constructed the first office of fingerprints in Basra. They applied Henry's method for the identification process. After that, this office was transferred to Baghdad City and named "Criminal Identification Bureau" under the supervision of Englishmen and Indians. In 1934, some officers were sent to India in order to study the science of fingerprints, and then they came back to Iraq [29].

The aim of this study is to make and find an AFIS using computer programs to recognize fingerprints, because the Criminal Identification Bureau in Erbil is using a classical method to do this work although there are (8000) fingerprints in that department related to criminals.

\section{Fingerprint Patterns and their Features}

\subsection{Fingerprint Patterns}

Each fingerprint has one of three basic patterns: Loop, Whorl and Arch [27], [5].

$\left({ }^{*}\right)$ Biometrics: It is the science of measuring different aspects of the human body and provides near foolproof security because it relies on a person's unique makeup [24]. 
According to Edward Henry's classification, the fingerprint is classified into the following eight-subclasses [5]:

a. Arch: is sub-classified into:

1. Arch (A): It is the pattern in which the ridges enter in one side, rise in the middle, and flow or tend to flow out from the other side as shown in figure (2.1a).

2. Tented Arch (T): It is the pattern in which the ridge have the tendency to enter from one side and flow out from the other side, with the exception that the ridges from either an angle or an upthrust on the center. Figure (2.1b) shows a typical tented arch fingerprint.

\section{b. Loop: Its is sub-classified into:}

3. Right Loop (R) or Radial Loop: It is the pattern in which one or more of the ridges enter in either the side of the impression, recurve, touch or pass an imaginary line drawn from the delta to the core, and terminate on, or toward the same side of the impression from which such a ridge or ridges entered. Radial loops, as shown in figure (2.1d) must have a sufficient recurve, a delta, and a ridge count across a looping ridge; the proper location of the delta and core is extremely important in the classification of patterns. The ridges of radial loop flow towards the radius bone (thumb).

4. Left Loop (L) or Ulna Loop: It is exactly like the radial loops except the direction of the ridges which is toward the ulna bone (the little finger), see figure (2.1c).

\section{c. Whorl (W): It is sub - classified into:}

5. Plain Whorl: It is the pattern in which at least two deltas and one recurving ridge such as a spiral or any variation of a circle must exist as shown in figure (2.1e).

6. Central Pocket Loop: It is placed in the central pocket loop, which has two deltas and at least a ridge making a complete circuit as in the right loop, the imaginary line draw between the two deltas must not touch any of the recurve ridges within the pattern area.

7. Double Loop: There are two deltas and two separate loops with separate and distinct shoulders in each double or twinned loop.

8. Accidental Whorl: It is a pattern consisting of a combination of two or more different types of configurations with the exception of the arch.

But now several scientists classify FP's into five classes [1][13][26]: 


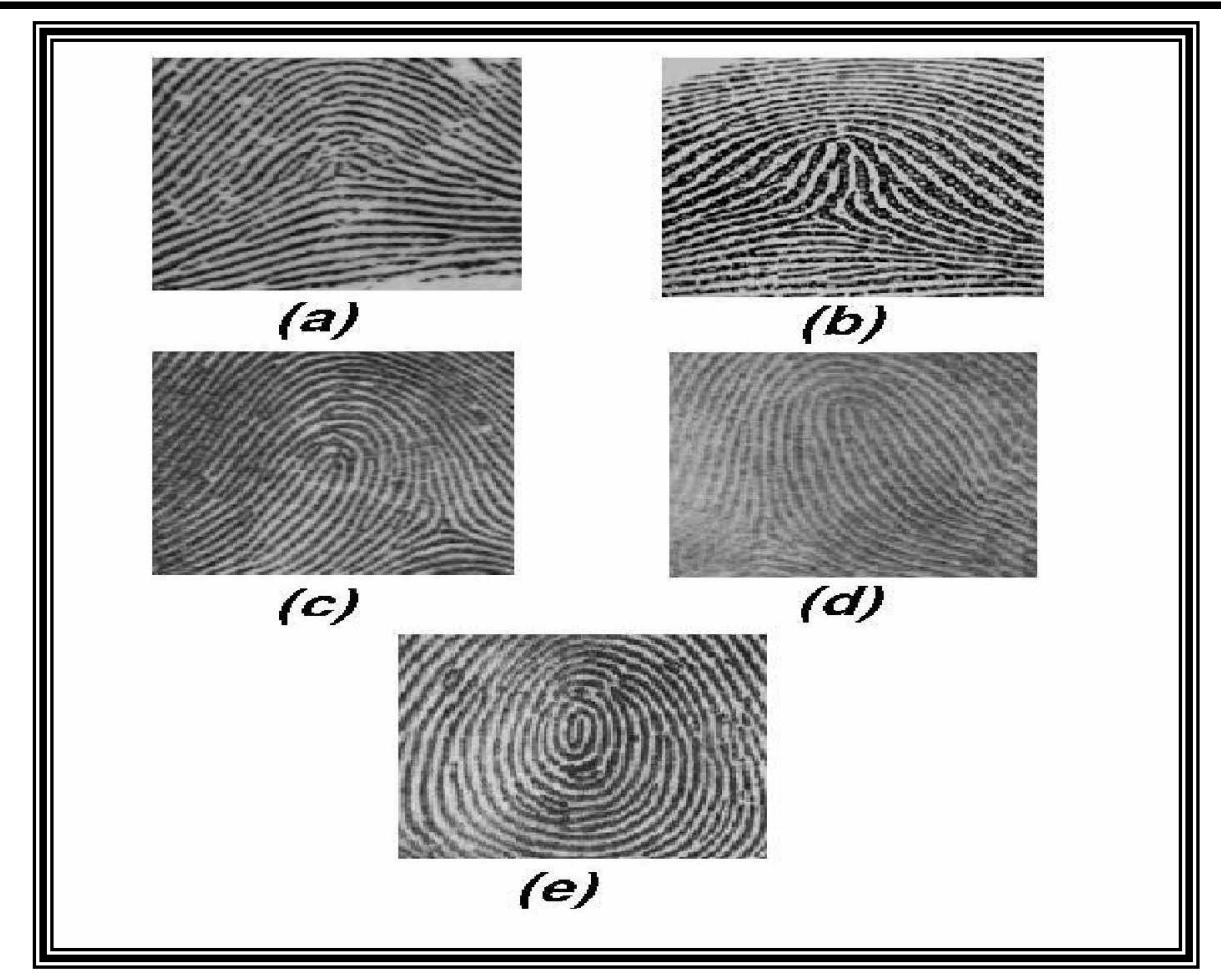

Figure 2.1 fingerprint patterns:
a- $\operatorname{Arch}(A)$.
c- Left Loop (L).
b- Tented Arch (T).
d- Right Loop (R).
e- Whorl (W).

Whorl (W), Right Loop (R), Left Loop (L), Arch (A), Tented Arch (T). For more illustration see figure (2.1).

\subsection{Fingerprint Properties}

Fingerprints have three important properties, as follows [5]:

1. Unchangeability: The ridges and details of FP's are constant through life until the skin disintegrates after death.

2. Uniqueness: There are no two fingerprints, which have the same pattern.

3. Classification: The variations of FP's are within a limit, which allow a systematic classification of the patterns.

\subsection{Fingerprint Features}

There are two main types of features in fingerprints used as an in identification of individuals [13]: 


\section{A. Global Features \\ B. They are those characteristics seen with the naked eye. The classifications of fingerprints are based on the global features, which include:}

\section{Pattern area}

It is the part of the fingerprint that contains all the global features. While, the pattern area of loops and whorls can be easily defined, it is very difficult to define the area of arch patterns.

\section{Core Point}

It is located at the approximate center of the finger impression.

\section{Delta}

It is the point on the first bifurcation, abrupt ending ridge meeting of two ridges, dot, fragmentary ridge, or any point upon a ridge at the nearest center of divergence of two type lines.

\section{Type lines}

They are the two innermost ridges that start parallel, diverge, and surround or tend to surround the pattern area. They are often breaking the ridge immediately outside that line is considered to be its continuation.

\section{Ridge count}

It is the number of ridges in the Pattern Area. To establish the ridge count, an imaginary line is drawn from the Delta and the Core and each ridge that touches this line is counted.

\section{B. Local Features}

They are Minutia* points. It is possible for two or more individuals to have identical global features, but they still have different and unique fingerprints, because they have local features (minutia points):

There are five different characteristics of minutia points in fingerprints:

1. Type- There are a number of types of Minutia points such as:

A. Ridge Ending: It occurs when a ridge ends abruptly, see figure (2.2A).

B. Ridge Bifurcation: The point at which a ridge divides into two or more branches, see figure (2.2B).

C. Ridge Divergence: The spreading apart of two lines which has been running parallel or nearly parallel, see figure (2.2C).

D. Dot or Island: A ridge that is so short, it appears as a dot, see figure (2.2D).

E. Enclosure: A ridge which is divided into two and then reunites again creating an enclosed area of ridge -less skin, see figure (2.2E).

F. Short Ridge: An extremely short ridge, but not so short and appears as a Dot or Island, see figure (2.2F).

2. Orientation- Each Minutia point faces in particular direction.

$\left(^{*}\right)$ Minutia points are the points at which ridges end, fork and change. 
3. Spatial Frequency- Spatial frequency refers to how far apart the ridges are in the neighborhood of the minutia point.

4. Curvature- refers to the rate of change of ridge orientation.

5. Position- The position of the minutia point refers to its $\mathrm{x}, \mathrm{y}$ location.

Figure (2.2) Local Features:

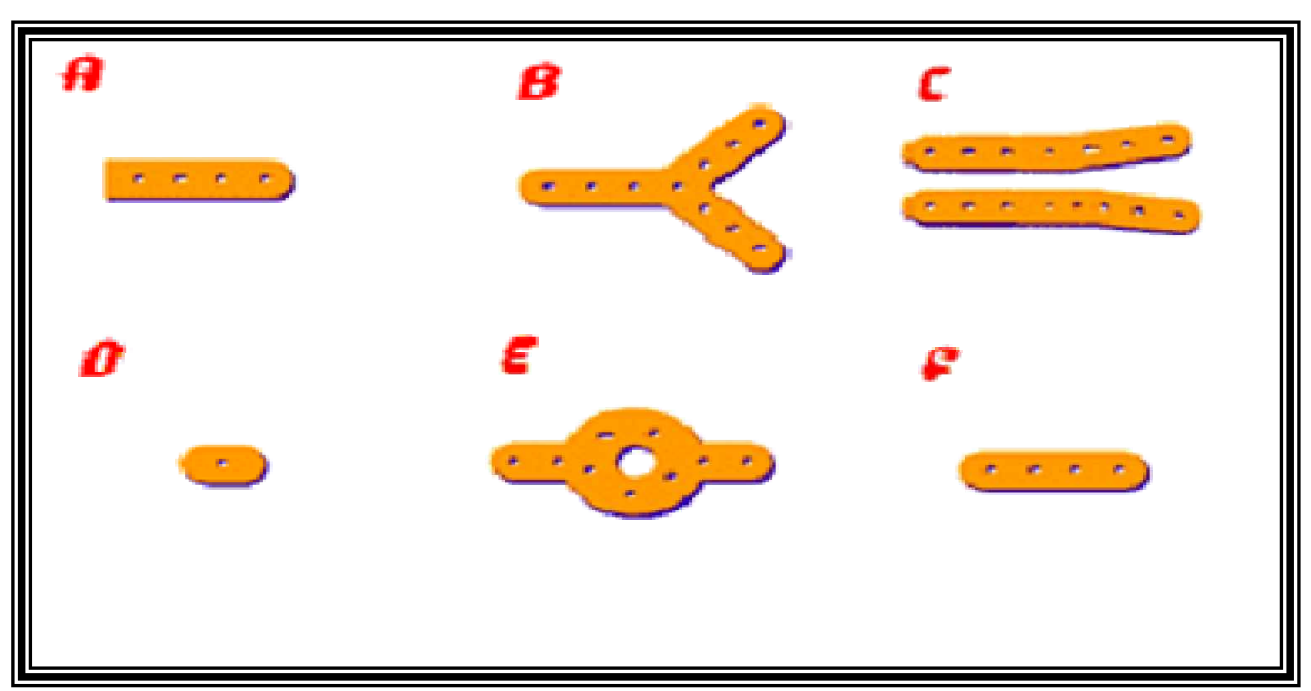

Figure 2.2 Local Features:
A- Ridge Ending.
D- Dot or island.
B- Ridge Bifurcation.
E- Enclosure.
C- Ridge Divergence
F- Short Ridge.

\section{Computer Imaging}

Computer imaging is one of the important subjects nowadays; it deals with digital images including the Acquisition, Enhancement, and Recognition of images. Computer imaging can be defined as the acquisition and processing of visual information by computers [27].

There are two major kinds of computer imaging [27]:

1. Computer Vision: In computer vision the computer uses applications of the processed images.

2. Image Processing: In image processing applications of the output images are for human use.

Computer imaging has many applications in medicine, industry, printing and publishing, cosmetics and personal grooming, astronomy, mineral analysis, fields of law enforcement and security and so on [18].

\section{Expert systems [9]}

Expert systems have been used successfully in various applications, ranging from image processing and industrial inspection to the medical diagnoses and process control. Expert systems are human-machine systems 
with specialized problem solving expertise. Thus, the name expert system reflects the fact that these systems are generally based on knowledge obtained from people who are experts in a specified discipline.

\section{Classification}

Sometimes the recognition process involves much time to do the operation of recognition. But another important procedure is to reduce the time of recognition, by partitioning the entire data to some classes by classification process.

The pattern classification, as related to image analysis, involves taking the features extracted from the image and using them to classify image objects automatically [23].

For a two-class pattern classification problem, there are the hypotheses:

$\mathrm{H}_{0 \text { : }}$ The pattern belongs to class one.

$\mathrm{H}_{1}$ : The pattern belongs to class two.

If there are more than two classes, $\mathrm{H}_{\mathrm{k}}$ is the hypothesis, which assumes that the pattern belongs to class $\mathrm{k}$. A typical example of multiple hypothesis testing is the classification of fingerprints type, where types of fingerprints are equal to five [25].

\section{Data Collection Surces}

We prepared AFIS, software in Visual Basic, to distinguish between fingerprints. In order to implement AFIS, we need fingerprint data; the required data were not available as mentioned before in "Erbil Criminal Identification Bureau", data was acquired from Bologna University in Italy. Those fingerprint images were taken from left thumb finger and corresponding to (21) persons ( 8 items per person). The type of all those fingerprints is (JPEG) and $(256 \times 256)$ pixels. In addition, (50) fingerprints were taken from left thumb finger corresponding to (25) persons ( 2 items per person) by a manual method, using a scanner of type (48) bit. These two fingerprints are selected from four impressions (items) of the same person in order to obtain good images in all directions.

For fingerprint purchasing refer to (www.nist.gov).

\subsection{Low Level Processing}

The processing of algorithm used was as follows (figure, 7.1):

1. Choose a fingerprint image.

2. IF the size of the image is equal to $(200 \times 200)$ pixels

THEN GO TO the step 6

END IF.

3. Shrinking or stretching.

4. Showing the image in the left picture box.

5. Copying the fingerprint image.

6. Transforming the image's copy from color type to grayscale type. 
7. Deleting the first and last 20 rows and columns from the image's copy.

8. Showing the image in the right picture box.

9. Stopping.

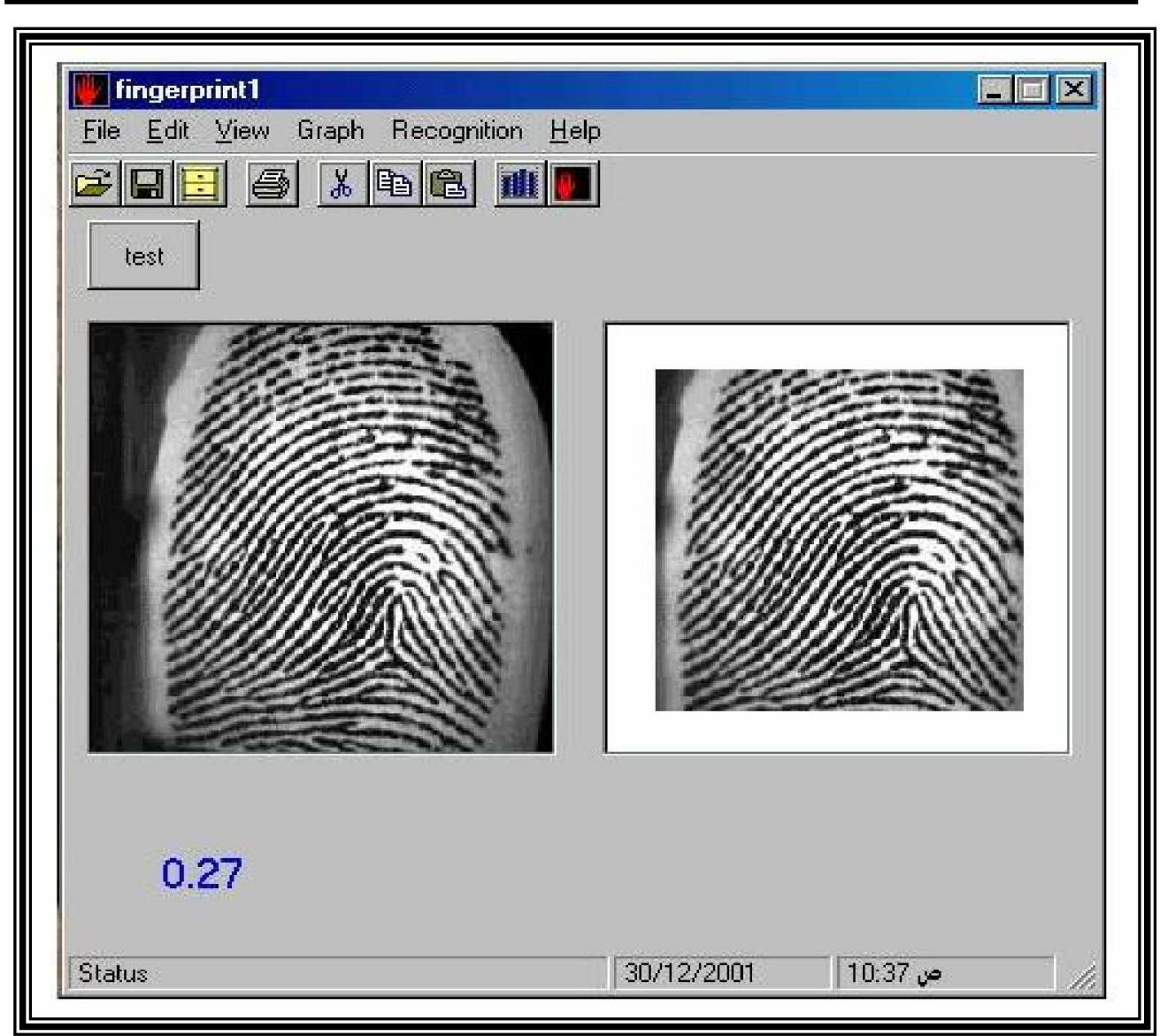

Figure 7.1 : Main form of the program

\subsection{Intermediate Level Processing}

In this stage, three processes were made; Segmentation, Representation and Description. The size of the image in the right picture box is reduced to $(160 \times 160)$ pixels. After that, these $(160 \times 160)$ pixels are divided into (64) squares; the size of each square is equal to $(20 \times 20)$ pixels (400 pixels per square). This is the segmentation process, figure (7.2).

Directly after segmentation process, the representation process comes. The internal characteristics of the image are chosen, therefore, the present values in each pixel are the raw data that we depend on to represent each square inside the fingerprint image. 
After that, the image was described by an approach, there are many approaches to describe the image, we have chosen statistical approaches. 8 parameters were used in this step, they are as follows:

1. Mean $(\mu)$

2. Median (Med).

3. Quadratic mean $(\mathrm{Q})$.

4. Harmonic mean $(\mathrm{H})$.

5. Variance (Var).

6. Skewness ( SK)

7. Kurtosis (k).

8. Mean deviation (Mean D.)

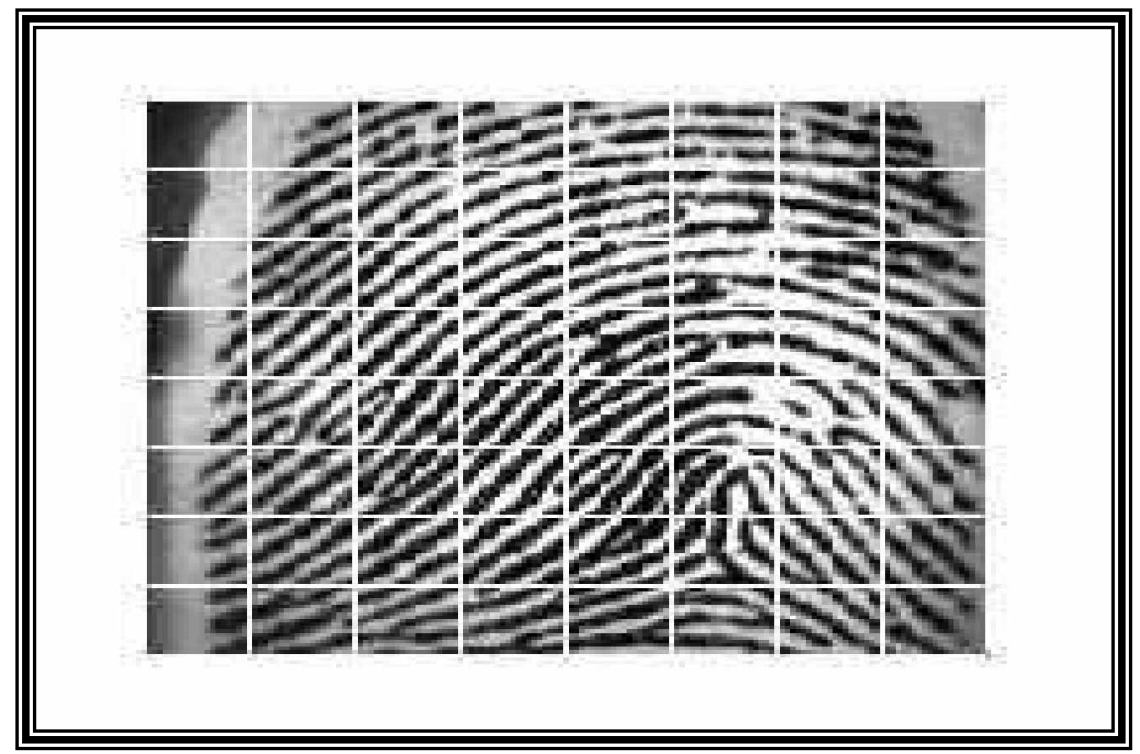

Figure 7.2: Segmented fingerprint

\subsection{High Level Processing}

The final step in AFIS is the recognition of fingerprint images. A robust distance is applied to compute the distance between parameters for each two squares in the same location but for two different fingerprints. The first one is fingerprint to be recognized, and the second one is the stored fingerprint in the storage unit of the computer. This operation (distance between two squares) is carried out for all (64) squares in the fingerprint image. The equation of robust distance measure is as follows:

$$
\mathrm{D}_{\mathrm{k}}=\frac{\sum_{i=1}^{8}\left|a_{i}-b_{i}\right|}{\sqrt{\sum_{i=1}^{8} a_{i} * \sum_{i=1}^{8} b_{i}}}
$$


Where:

$\mathrm{a}_{\mathrm{i}}$ : It is the ith parameter for the first square.

$b_{i}$ : It is the ith parameter for the second square.

After computing the (64) distances of the selected fingerprint to be recognized, all fingerprints would be stored in the storage unit. The minimum distances for each square from these (64) squares are computed, in order to know which fingerprint has a maximum number of squares and, at the same time, possess the minimum distances (i.e., the fingerprint that matches the unknown fingerprint must possess a great number of squares which have minimum distances). While, this operation is limited to the following:

If the number of squares that have minimum distances is less than (10), the matching process must be rejected. The result of recognition process is failure and the AFIS gives a message that the name of that person is unknown (the fingerprint is unrecognized). If the number of squares has minimum distances greater than or equal to 10 , it will lead to a message appearing in the message box telling the user of the name of the person, who possesses the fingerprint. Depending on the references [20] and [28], we have written this subroutine concerning the third current stage of image analysis.

In addition to robust distance, many measures are applied to find the distance between two images, such:
a. ED.
b. Chebychev Distance.
c. Manhattan Distance.
d. Canberra Distance.
e. Minkowski Distance. When $\lambda$ is equal to 3,4,5,6,7,10,20.
f. Mahalanobis Distance.
g. Similarity Measure.
h. Correlation.

In order to know which of the above measures the best is, we wrote a specific subroutine to compute them. Clicking the button (test) on the main form of AFIS leads to obtain the success rate of the particular measure used in the recognition process. Therefore, in each trial, we can change the measure and click the "test" button to know the best measure. The robust distance overcomes all other measures and obtains the percentage of succeeding equal to $(84.5 \%)$. On the other hand, Mahalanobis Distance came in the second order with percentage of succeeding equal to (82\%).

\section{Conclusions}

1. Using a specific scanner for acquiring fingerprint images leads to increasing the accuracy of FPR compared with the classical methods for acquisition the fingerprint images. 
2. Segmentation of the region in the fingerprint image by the researcher's method led to simplify the describing and recognizing processes, in addition to increase the resolution (accuracy) of the process.

3. The robust distance of calculating the distance between the two vectors has a good role in finding the minimum distance between two fingerprint images. The success rate of matching was reaching $(84.5 \%)$.

4. Expert systems are powerful methods in matching fingerprints, because they depend on simple procedures, and give excellent results.

5. The average time of completing the analysis process from acquisition step to a final step of intermediate level processing is equal to $(0.33)$ seconds, and the average time of recognition and interpretation is equal to (0.28) seconds.

\section{Recommendations}

1. The use of (AFIS) in "Erbil Criminal Identification Bureau"

2. The conducting of another study on the classification of fingerprint classes to reduce the time of recognition

3. Producing or buying a specific scanner for acquiring fingerprint images to the display unit of the computer directly.

\section{References}

1. Ballan, M., Sakarya, F.A., and Evans, B.L., " A Fingerprint Classification Technique Using Directional Images", Proceedings IEEE Asilomer Conference on Signals, Systems, and Computers, Nov 1997, Pacific Grove, CA, (USA), available on-line at http://www.ece.utexas.edu/ bevans/papers/1997/fingerprint/fingerprints.pdf

2. Chatfield, C., Collins, A. J., Introduction to Multivariate Analysis, Chapman and Hall, 1980 .

3. Chang, D.H., "Fingerprint Recognition Through circular sampling, 2001, available on-line at http://www.cis.rit.edu/ dxc.331/web_thesis/thesis.html

4. Duda, R.O. and Hart, P.E., Pattern Classification and Scene Analysis, John Wiley \& Sons, 1973.

5. Fu, K.S., Syntactic Pattern Recognition: Applications, Springer-verlag, 1977.

6. Fu, K.S., Digital Pattern Recognition, $2^{\text {nd }}$ edition, Springer-verlag, 1980.

7. Gonzalez, R.C. and Tou, J.T., Pattern Recognition Principles, Addison-wesl, 1974.

8. Gonzalez, R.C. and Thomason, Syntactic Pattern Recognition Principles: An Introduction, Addison-wesl, 1978.

9. Gonzalez, R.C. and Woods, R.E., Digital Image Processing, Addison-wesl, 1992.

10. Gordon, A., D., Classification, Chapman and Hall, 1981.

11. Gomes, J. and Velho, L. , Image Processing for Computer Graphics, Springer-verlag, 1997.

12. Halvorson, M., Microsoft Visual Basic 6.0 Professional, Microsoft press, 1998.

13. Jain, A.K., Prabhakar, S. and Hong, L., "A Multichannel Approach to Fingerprint Classification", IEEE Transactions on Pattern Analysis and Machine Intelligence, Vol. 21, No. 4, pp. 348-359, 1999.

14. Jain, A.K., Bolle, R. and Pankanti, S., Biometrics-personal Identification in Networked society, Kluwer Academic, 1999.

15. Keogh, E., "The History of Fingerprinting", 2001, available on-line at http://www.ics.uci.edu/ eamonn/cosmos.html 
16. Killer, A.M., A First Course in Computer Programming Using Pascal, McGraw Hill, 1986.

17. Koffman, Elliot, B., Turbo Pascal, Addison-Wesley, $5^{\text {th }}$ edition, 1995.

18. Niblack, W., An Introduction To Digital Image Processing, Prentice-Hall, 1986.

19. Pal, S.K., Fuzzy Mathematical Approach to Pattern Recognition, John Wiley \& Sons, 1980.

20. Petroutsos, E., Mastering Visual Basic 6, Sybex Inc., 1998.

21. Prince, A., Rinaldi, D. and Brirli, E., Visual Basic 6: How To, Sams publishing, 1999.

22. Roddy, A.R and Stosz," Fingerprint Features-Statistical and system Performance Estimation", IEEE, Vol. 85 No 9, 1997, pp. 1390-1421.

23. Umbaugh, S.E., Computer Vision and Image Processing: A Practical Approach Using (CVIP) Tools, Prentice-Hall, 1998.

24. White, Ron, "Biometric ID Systems", PC Computing, Vol. 13 Issue 1, Jan 2000, pp. 222.

25. Yaung, T.Y. and Calvert, T.W., Classification Estimation and Pattern Recognition, Elsevier, 1974.

26. Yao,Y., Fransconi, P. and Pontil, M., "Fingerprint Classification with Combinations of Support Vector Machines", Proceedings $3^{\text {rd }}$ International Conference on Audio and Video Based Biometric person Authentication, Sweden, June 2001, available on-line at http://www.dsi.unifi.it/ paolo/ps/avbpao1-finger-svm.pdf

\section{المراجع باللغة العربية}

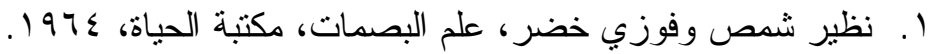

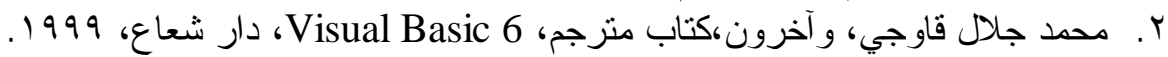

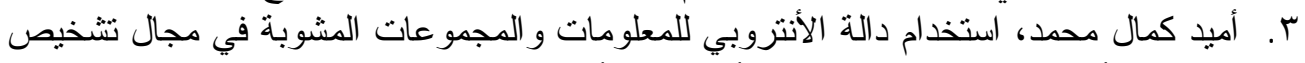

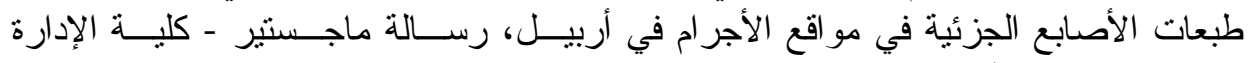

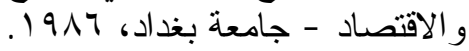

